\title{
SIMULATION OF HIGH RE BOUNDARY LAYER FLOWS USING VORTICITY CONFINEMENT*
}

\author{
JOHN STEINHOFF ${ }^{\dagger}$, SUBHASHINI CHITTA $^{\ddagger}$, AND YONGHU WENREN ${ }^{\S}$
}

\begin{abstract}
We describe how Vorticity Confinement (VC) can be regarded as a new pde formulation of the slightly viscous incompressible flow equations. These equations, when discretized and solved, generate nonlinear solitary waves that can be used to efficiently approximate a large class of external flow problems, including the effects of separating turbulent boundary layers. These problems can involve subsonic flow over complex structures such as ships, buildings, and realistic topography such as hills. These problems typically involve the simulation of a large ensemble of flow conditions for each configuration to be designed or analyzed. One of the most difficult aspects of these simulations is that often the main effects of the dynamics of thin evolving vortical structures must be solved for.

The VC method appears to be effective for many of these problems, since it requires much less computing and setup time than current Navier Stokes "RANS" approximations. The method involves treating the flow over a solid body as a two-scale problem: The first component is an "outer" smoothly varying, mainly irrotational flow with perhaps large scale vortical components where standard CFD techniques can be used. The second component is composed of thin vortical regions. These vortical parts consist of mostly thin attached boundary layers, thin separating vortex sheets, which roll up and thin vortex filaments, which result from roll up. The VC method involves treating these regions with a single equation that has three equilibrium states corresponding to these regions. The equation allows transition between these equilibrium states so that for example, boundary layers can separate and roll up into vortex filaments, and vortex filaments can join and reconnect with other filaments. These properties survive discretization and require no extra logic.

$\mathrm{VC}$ can be used to treat the entire flow in a locally-Cartesian computational grid with the solid surfaces "immersed" in the grid so that they can be quickly generated for many configurations. Adaptive or conforming fine scale grid cells are then not required to approximate the thin vortical boundary layers, or thin separating vortex sheets. Instead, vortical structures created with Vorticity Confinement, which are essentially thin, non-diffusing, or confined "Nonlinear Solitary Waves" (NSW's) are used to "carry" the vorticity in these regions. The VC method has the efficiency of panel methods, but the generality and ease of use of fixed grid Euler equation methods. In this paper we concentrate on attached and separating boundary layers; there are already in the literature a large number of papers describing the use of $\mathrm{VC}$ for free, convecting vortices.
\end{abstract}

Key words. Vorticity confinement, vorticity, vortex dominated flow, computational fluid dynamics, boundary layer separation, solitary waves, permanent wave.

AMS subject classifications. 65-XX, 75-XX.

1. Introduction. This paper describes the use of Vorticity Confinement (VC) for efficiently treating flow over bodies with attached and separating boundary layers, in high Re incompressible flows. These problems include complex blunt bodies with thin shed vortex sheets and filaments, and thin attached boundary layers on solid surfaces, where "thin" simply means having cross section dimensions much less than the overall body. (This is done, of course, when inviscid solutions are used as approximations for the full flow, by neglecting displacement thickness effects for attached boundary layers). Our method allows this approximation to be extended to classes of separating boundary layers. Because these flows involve turbulence in the vortical regions, there is currently no ab initio method to treat them on current or foreseeable computers. In fact, even with the employment of coarse-grained approximate model

\footnotetext{
*Received February 16, 2013; accepted for publication December 10, 2013.

${ }^{\dagger}$ University of TN Space Institute, 411 B H Goethert Parkway, Tullahoma, TN 37388, USA (jsteinho@utsi.edu); and Wave CPC Inc., 14 Brook Hollow Lane, Windham, NY, USA.

‡Wave CPC Inc., 14 Brook Hollow Lane, Windham, NY, USA (subha@wavecpcinc.com).

§AMRDEC, Redstone Arsenal, AL 35898, USA (yonghu.wenren@us.army.mil).
} 
pde's for turbulent flows such as "RANS" or "LES", because of these thin regions there still is no conventional method efficient enough to adequately treat many of these problems, for routine design/analysis of general configurations. As a result, in spite of years of turbulence modeling efforts in CFD simulations, serious flaws in aerodynamic design of large scale systems involving vortex shedding may still be left undetected until the expensive operating prototype or production stage.

For these reasons, the VC method should help find vorticity induced aerodynamic problems by rapidly simulating large numbers of operating conditions, as is often done in much more expensive testing programs for flying prototypes, and in realistic simulations. In this way it is different from conventional viscous CFD methods where much of the computational effort is often devoted to calibrating and attempting to resolve model pde's in thin turbulent regions with complex, fine, body-fitted grids. Such conventional methods can be useful for simulating a small number of pre-defined flight conditions (or vehicle designs), where each simulation may require high computational cost, but may miss isolated, critical vortex-induced problems.

Our basic premise is that, for a class of real-world problems requiring simulating ensembles of flow conditions, we are not necessarily guaranteed higher accuracy overall solutions by using large amounts of computing time and memory in employing conventional turbulence models (both for boundary layers and wake), when by doing so we can only solve for a small number of cases due to cost constraints. To achieve dramatically lower computational cost, $\mathrm{VC}$ uses moderately coarse grids to solve for the inviscid part from known first principles, but also uses simple models to solve for the small scale turbulent parts, on the same grid.

We only consider single phase incompressible flow, hence there are no shocks or fluid/fluid interfaces and the vortical regions mentioned are the main regions with large velocity gradients. As such, they represent the main sources of difficulty in computational simulations. However, computing them can be very important since they can have a large impact, for example, if they are shed from a boundary layer and subsequently interact with other surfaces. In fact, as stated above, they have been implicated in a number of important aerodynamic design flaws, which could not be simulated during the initial design phase and were not found until the prototype or production stage.

To quote Wright [40], "It is actually a common situation in continuum physics that important physical processes occur at an inconveniently fine scale. Besides failure processes, other examples are turbulence and boundary layers in fluids..."

$\mathrm{VC}$ has features in common with other Eulerian methods developed for problems with thin features. These other methods were also developed in the last several decades: they are known as "diffuse interface", "solitary wave", "phase field", primarily for condensed matter physics and "shock capturing" in compressible fluid dynamics problems. VC, however, takes advantage of special features of vorticity, discussed below, that the other methods do not and can be thought of as an extension of the above techniques. These features allow VC to automatically capture discretized multidimensional vortical structures (attached, separating, or convecting) with cross-sections as thin as a few grid cells.

1.1. VC as an implicit vortical model. To achieve efficient computations and retain accuracy to realistically simulate main vortex effects, we ignore details of the internal structure of thin vortices and employ very simple implicit models, which are easily discretized on relatively coarse grids (with no numerical diffusion), instead of trying to approximate them with a detailed pde turbulence model, which would 
require much finer grids to compute. This basic approach has been successfully used in the past, but with more complex Lagrangian "vortex lattice" (or "panel") schemes, as described below.

Like VC, these past Lagrangian methods have also ignored internal details of thin vortical structures. This has been feasible because, as is well known, the local external velocity induced by a thin uniform vortex sheet or a typical vortex filament depends only (to a good approximation) on the total vorticity in a cross section and is independent of the internal details of the vorticity distribution [20]. This fact is important because otherwise the solutions would be sensitive to whatever model is adopted for the internal structure. This feature has been used in employing the BiotSavart law to model shed vortex sheets as sets of filaments [38] [24] since 1931, and for many decades by "panel methods [13]" or "integral methods" for both shed vortex sheets/filaments and solid surfaces with attached boundary layers.

These approaches, (as well as VC), are not necessarily less accurate than attempts at solving dynamical pde's for the internal details of the vortical distributions at high Re (such as common approaches to the Euler and Navier Stokes equations with eddy viscosity models), since the vortical flow then is usually turbulent and, of course, as stated, only approximate model pde's can then be formulated.

The main problem with these Lagrangian schemes is that (unlike VC), they treat vortical regions with sets of computed Lagrangian markers, which leads to computational complexity and cannot usually be generalized. These difficulties are also present in "shock fitting" schemes, leading to their (almost total) abandonment in favor of Eulerian "shock capturing" methods.

Most conventional Eulerian methods now in common use do not need Lagrangian markers, of course. However, the computed vortical distribution in a thin vortical region will then be dominated by discretization error on the Eulerian grid, which leads to an unrealistically very viscous solution unless a computationally expensive fine grid is used, and may not even qualitatively resemble the solution of the original model pde to be solved. In fact, the actual measure of many Eulerian solution efforts involving thin shed vortices with pde models seems to be to merely the ability to keep the thickness of convecting vortices from becoming large due to numerical error [23].

A further point is that even "high order" Eulerian schemes may not limit this error (for conventional discrete methods). This is because the "order" is only a meaningful estimate when the number of grid cells across the feature is large. Unfortunately, many complex problems have numbers of convecting vortices or boundary layers, and cannot have a large number of cells across each of the many cross sections, for feasible computational grids.

An important feature of $\mathrm{VC}$ is that it results in a controlled, simple computational model for the vorticity distribution, as opposed to conventional Eulerian CFD schemes, which allow the error to grow to an uncontrolled mixture of discretization errors. This is true even though $\mathrm{VC}$ also solves a pde, which is also discretized on an Eulerian grid. As such, VC is an "Implicit nonlinear modeling" technique, which has a well-defined solution, "sampled" on the grid, while solutions with conventional CFD schemes for thin regions will be a result of an under-resolved discretization scheme, unless a very fine grid is used. For this reason, for VC, the resulting vorticity distribution can then be classified as a well-defined "Nonlinear Solitary Wave" (NSW).

To quote Bishop, et. al. [2], "The central point is that solitary wave solutions then provide a starting basis for a configurational phenomenology which is vastly more realistic than anything attainable by conventional perturbation (we would add linear 
CFD) theory."

Reviews of research on Vorticity Confinement appear in books [33] [26] [28] [11], as well as a large number of papers (a few include references [22] [21] [12] [5] [1]), mostly on free convecting vortical wakes.

VC was originally developed (in 1990) for simulating free vortex wakes, independently of work on solitary waves. Several years later (1997), VC was implemented for simulating flow over sold bodies, with thin vortical boundary layers. This development repeated the earlier cycle involving: first, Lagrangian filament simulations for convecting vortices (in the middle of the last century); then, simulations of free vortex wakes. Subsequently, sets of filaments were used to simulate flow over solid bodies (as "vortex lattice", or "panel" methods).

1.2. Outline of paper. We emphasize that the NSW's are basic mathematical structures resulting from the pde's. In this way, $\mathrm{VC}$ can be considered to be a qualitatively new formulation of the slightly viscous, incompressible flow equations. As explained, it should be simpler to use this approach as both an efficient way to get approximate solutions and as a "starting point" to build refined models for the boundary layer (BL). This approach should be far more effective than using the usual continuum Navier Stokes equations with an eddy vorticity model, discretized on a body-fitted grid with an extensive boundary grid refinement, as a starting point for more refined boundary layer computations. The main concept is that NSW's can have three equilibrium states: attached boundary layers, free convecting vortex sheets and vortex tubes. The transitions between these states are determined by basic flow equations.

1. First, the basic idea of using a simple, nonlinear pde structure to model thin attached vortical boundary layers will be discussed. We define these structures as the basic NSWs of the flow. This solution is first described for BL's that are aligned with a grid plane, where imposition of boundary conditions is simple. A vital point is that these NSW's do not impose a flow profile, but attract it, so that the BL can temporarily deviate, and, for example, separate.

2. We show how these NSW's can easily be created, first as a solution to a pde, then as a finite difference solution, discretized on a locally uniform, but not necessarily aligned with the grid. These NSW's can be constructed along solid surfaces to enforce no-slip boundary conditions and as simple efficient models for thin boundary layers which do not require fine "viscous" grids. When used with any inviscid "outer" flow solution, these NSW's can model the full flow in a very simple way for many cases.

3. We then show how these NSW's can separate and be captured as convecting thin structures, while requiring no special logic. This separation occurs automatically at discontinuities in surface angles and in localized regions of large adverse pressure gradient. - (Separation on smooth surfaces may depend on the internal states of the turbulent boundary layer, and require extensions to this model, which is also true of all other BL models). For flows with separation from smooth surfaces, VC (NSW's) can then serve as an efficient "starting basis", as suggested by Wright in the above quote, since the simple VC method described here, alone, results in qualitatively accurate flows for many cases, with no additional parameters or extra terms, on relatively coarse grids.

4. The ability of these NSW's to convect with the flow as free vortices and to 
change topology, such as separating, rolling up or reattaching, while still obeying the same nonlinear pde will be discussed. When the pde is discretized, the same basic method can be used in the boundary layer and separated region, independent of the topology, and complex logic can be avoided. After separation, these convecting NSW's confine vorticity along gradients of vorticity magnitude (towards the vortex centers). In this way, they can relax to and "carry" vortical structures and can simulate dynamics of freely convecting vortex sheets and filaments. This simulation includes merging and reattachment, with no extra logic, because, like the BL, the vortex structure, is not rigidly prescribed, but quickly relaxes to an equilibrium state (over a few time steps). NSW's can also serve as models for physical convecting vortical sheets, where approximations to physical features such as Kelvin Helmholtz instabilities are automatically incorporated, without damping effects typically due to discretization error. (Unlike these free vortex NSW simulations, the boundary NSW's defined in sections 2 and 3 confine vorticity towards a predefined solid surface to approximate (possibly separating) boundary layers.) The discussion of convecting vortices is brief, since many papers have already been written on applications of $\mathrm{VC}$ to this subject, as can be seen in references [33] [26] [28][11] [10] [30] [27].

5. Finally, a number of cases are referred, which have been demonstrated and published. These range from 3-D flows over disks, cylinders and parachute wakes, to complex flows produced by configurations such as rotorcraft, including brownout. New results will be shown for vortex induced separation from attached boundary layers as well as (tripped) boundary layers on a solid surface that is not aligned to the grid.

An important feature of $\mathrm{VC}$ is that it can be used with many existing subsonic flow solvers. It has also been used for supersonic flow [23]. Any numerical diffusion from these solvers can be compensated for by VC. For this reason, a rough estimate of this numerical diffusion must, of course, be available to be used by VC. Alternatively, if the entire solver, including convection, were written from the beginning, including VC as a self-contained program, this estimate would not be needed, but much flexibility would be lost.

2. A simple model for nonlinear solitary wave (NSW) generation on boundaries aligned with the grid. As a simple illustration, we first consider a small section of the flow over a surface and assume that the "outer" irrotational flow has no tangential pressure gradient, and that the outer tangential velocity in this region is independent of streamwise position $(x)$. Variations in the BL velocity, which lead to separation, will be considered later. We also (at first) assume that the normal velocity $(v)$ is zero. We then have a simple, 1-D model for the tangential velocity in the boundary layer, $u(\mathrm{y})$. The boundary conditions are $u(0)=0$ and $u(Y)=U$, where $Y$ is the thickness of the BL (outer edge), and $U$ is the (inviscid, irrotational) tangential velocity at the outer edge of the BL. We assume that $u$ is given by an "outer" inviscid solution of the Euler equations. No grid interface is required since the velocity will automatically approach this velocity.

We first make this problem look like a very simple convection - diffusion problem. This solution will have all the salient features of the general flow (even with nonaligned boundaries) described in the next section, but is much easier to understand. Our treatment will involve boundary layer vorticity (rather than fluid) convecting towards a solid surface, balanced (after relaxation) by diffusion transporting vorticity away 
from the surface. The vorticity then forms a stable, finite thickness boundary layer. The convection is by a constant artificial velocity $(w)$, toward the surface. At the same time, vorticity is diffusing away from the surface with constant diffusion constant $(D)$. (This normal diffusion can also model numerical error.) The dynamics describes the (one dimensional) boundary layer model, as a function of the normal distance to the surface $(y)$. The governing pde is

$$
\partial_{t} u(y, t)-w \partial_{y} u=D \partial_{y}^{2} u .
$$

The diffusion in this simple model is only in $u(y)$. As stated, the "confinement" term $\left(-w \partial_{y} u\right)$ serves to convect vorticity toward the surface. Then, after a small number of time steps, the steady state asymptotic solution for $u(y)$ is approached:

$$
u=U\left(1-e^{k y}\right)
$$

where $k=\frac{w}{D}$ and $w$ is negative. This results in a vortical layer confined to the surface. When discretized, VC can result in effectively a 2-3 cell thick, stable boundary layer. At steady state, $\partial_{t} u \rightarrow 0$, the corresponding second order discretized equation is

$$
D \delta_{y}^{2} u_{j}=w \delta_{y} u_{j}
$$

where $\delta_{y} u_{j}=\frac{u_{j+1}-u_{j-1}}{2 h}, \delta_{y}^{2} u_{j}=\frac{u_{j+1}-2 u_{j}+u_{j-1}}{h^{2}}$ The discretized form now is

$$
u_{j+1}-2 u_{j}+u_{j-1}=\frac{w h}{2 D}\left(u_{j+1}-u_{j-1}\right) .
$$

Substituting the solution, $u_{j}=U\left(1-e^{j \alpha h}\right)$, in the above equation, we get,

$$
\begin{gathered}
e^{\alpha h}-2+e^{-\alpha h}=\frac{k h}{2}\left(e^{\alpha h}-e^{-\alpha h}\right), \\
e^{\alpha h}=\left[1+\frac{k h}{2}\right]\left[1-\frac{k h}{2}\right]^{-1} .
\end{gathered}
$$

The important point is that the constant in the exponential $(\alpha)$ can be increased until the main region of confinement is as small as one or two cells and the solution will remain stable. Thus, there will be no need to have a fine boundary layer grid to reduce numerical dissipation (due to numerical advection of the tangential velocity), just to simulate a thin BL profile, if only integral properties are to be modeled. An important point is that this BL is not specified, but relaxed to a given profile. Then, an integral BL model can be implemented so that the layer can respond to the dynamics and remain thin, become thick, even change topology and separate. (Detailed modeling can be accommodated by allowing $k$ to vary.) This will serve as a prototype for a turbulent BL model when applied to separation on smooth surfaces. An important feature is that the inward vorticity convection is effected by simple velocity acceleration tangential to the surface, which does not violate the boundary condition for zero normal velocity at the surface, or zero velocity divergence for incompressible flow.

Harten [8] tried to confine vorticity by altering the characteristics in a compressible computation. Even though vorticity is convected by the flow, changing only the characteristics will convect fluid as well as vorticity, which will alter the mass balance. For incompressible flows, our main concern, the pressure correction would then lead to an added velocity each time step along the vorticity gradient that would cancel the imposed modification of the characteristics. By contrast, our modification involves only a tangential acceleration and has no direct effect on mass balance. 
2.1. Physical interpretation of $\mathrm{BL}$ treatment. A physical interpretation of $\mathrm{VC}$ here involves simulation of an attached, turbulent boundary layer. If a BL is laminar and not yet turbulent and, if there is no pressure gradient, the boundary thickness will grow as the square root of downstream distance due to viscous diffusion. However, after transition to turbulence, the "viscous sub layer" where much of the velocity gradient occurs, has only a very small growth rate in thickness. This can be modeled as some of the outer tangential flow being directly transferred to the top of the sub layer by large scale vortices and accelerating it rather than gradually diffusing through the BL. This then has the same features as VC and results in the same constant thickness of the sub-layer.

If the $\mathrm{BL}$ separates, $\mathrm{VC}$ will continue to confine the convecting vorticity along gradients of vorticity magnitude. When a separated vortex sheet rolls up and forms an approximately axisymmetric vortex, this confinement will then be towards the vortex center. In this way, separating vortical structures and dynamics of freely convecting vortex sheets and filaments can both be automatically simulated with the same method. This simulation includes merging and reattachment, with no extra logic, because, like the BL, the vortex structure, is not rigidly prescribed, but transitions and relaxes to another equilibrium state (over a few time steps). NSW's can also serve as models for physical convecting vortical sheets, where approximations to physical features such as Kelvin Helmholtz instabilities are automatically incorporated. (Unlike free vortex NSW simulations, the boundary NSW's confine vorticity towards a predefined solid surface to approximate boundary layers.)

Thus NSW's, constructed along solid surfaces to enforce no-slip boundary conditions, can also serve as simple efficient models for thin attached boundary layers, even when not aligned with the grid, as described below. When "immersed" in an inviscid "outer" flow solution, these NSW's model the viscous ("RANS") boundary layer structure in a very simple way. As stated, these boundary layers can also separate and be captured as convecting thin structures, while requiring no special logic. This separation will also occur automatically at discontinuities in surface angles and in localized regions of large adverse pressure gradient.

3. Immersed boundaries. Solid wall boundary conditions can, of course, be very easily implemented if the wall lies on a coordinate surface. However, we can also easily enforce solid wall boundary conditions on immersed surfaces not aligned with the grid using the VC method: First, the surface is represented implicitly by a smooth contour of a function, defined at each grid point in a non-fitted locally Cartesian grid. This function can be just the (signed) distance from each grid point to the nearest point on the surface of an object. Then, at each time step when the discrete equations are being solved, velocities in the interior are simply reduced to zero with distance to the surface, and VC is used to "confine" the resulting vortical boundary layer. In a computation without VC, the layer would grow in the streamwise direction due to numerical diffusion. With $\mathrm{VC}$ (discussed in section 4), when diffusion is (automatically) balanced, the result will be a thin constant vortical BL region along the surface (if the outer tangential flow is constant), which is smooth in the tangential direction (because of the effectively tangential diffusion), with no accumulation of error due to numerical effects (as shown in Figure 1). (Note that the numbers on the ordinates of all the figures denote the normal distance (height) in terms of grid cell units.)

An important point is that no special logic is required in the "cut" cells, unlike many conventional immersed surface schemes. Further, many conventional immersed 
surface schemes must be inviscid because of cell size constraints (near the "surface", if the grid is Cartesian, very thin nonaligned grid cells cannot be used). With VC, there is effectively a no-slip boundary condition, which results in a boundary layer with well-defined total vorticity which remains thin (as an NSW), even after separation. This can be accomplished because numerical spreading (diffusion) can be eliminated without requiring long thin cells along the boundary, which would otherwise tend to spread a separating thin vortex sheet in the tangential direction.

4. Vorticity confinement (VC) for general flows. The basic principle of VC, as in the one-dimensional scalar example described in section 2 with convection is that the solution is generated as a stable structure that can be propagated indefinitely with no numerical dissipation but with a structure that is not rigidly specified, but can change topology (due to convection): There can be a number of "attracting" solutions that the solution can relax to and still obey conservation laws. These include, besides attached translating BL's, separated vortex sheets and rolled up vortex tubes. This feature allows the BL to separate in an adverse pressure gradient in a realistic way. Although the $\mathrm{VC}$ equations can be written as a discretization of a pde, the resulting solution at the small scales (within the structure) is not, of course, meant to be an accurate or even approximate solution of the original pde. This is because VC, as stated, is meant to capture, or model the small scale features over only a couple of grid cells, so that the discretization "error" is $\mathrm{O}(1)$ there. As explained, the basic goal is to simulate the qualitative effects of the BL on the outer flow in a very simple way.

\subsection{Convecting structure simulation.}

4.1.1. PDE representation. As a demonstration of the mathematical basis of the $\mathrm{VC}$ concept for convecting vortices, we first consider a passive scalar, $\phi$, advecting in 1-D at a constant speed $c$ :

$$
\partial_{t} \phi=-c \partial_{x} \phi
$$

Our basic point is that there will be errors when we discretize equation (7) using conventional schemes based on Taylor expansions. These errors will grow over time with linear discretization. If we confine a short "pulse-like" solution to $2-3$ grid cells, which is our goal, the derivatives of $\phi$, and hence the numerical "errors" will then become large. Corresponding to the small number of grid points within the pulse, there will be only a small number of quantities that we can conserve. This will meet our goal, if we can eliminate accumulating numerical error, and keep the pulse from spreading or diverging, since our goal is only to propagate a few conserved variables. For a scalar, the important quantities will be the total integral of the scalar and the position of the centroid. For a vortex filament (in 2-D or 3-D), the important quantities will include the total circulation around each cross section as well as centroid location.

The simplest method would be to use an "internal" convection on either side of the pulse peak. This would involve a balance between constant inward "confinement" transport and diffusion:

$$
\partial_{t} \phi=-c \partial_{x} \phi-\varepsilon \partial_{x}\left[\operatorname{sgn}\left(\partial_{x} \phi\right)\right] \phi+\mu \partial_{x}^{2} \phi .
$$

This would conserve $\phi$, but not exactly conserve the convecting speed of the centroid. This could be useful as an approximation. But first, we describe a method that exactly conserves the speed of the centroid. 
By simply adding a term, $\partial_{x}^{2} F(\{\phi\})$ to equation (7) that vanishes at the boundaries, along with sufficient derivatives, we can easily achieve our goal. This extra term will not affect the conservation of quantities of interest, which include the total amplitude

$$
A=\int \phi d x
$$

and the speed of the centroid

$$
\frac{d\langle x\rangle}{d t}=\frac{\int \phi c(x) d x}{A},
$$

where the centroid is, $\langle x\rangle=\frac{\int x \phi d x}{A}$.

We then have

$$
\partial_{t} \phi=-c \partial_{x} \phi+\partial_{x}^{2} F .
$$

To preserve the essential physics of equation (7) for a short convecting pulse, we want $F$ in equation (11) to satisfy an important condition: It should be homogenous of degree one, like equation (7), so that it does not depend on the magnitude of $\phi$. This is an important distinction of the VC equation. Many similar "phase field" nonlinear equations use non-homogenous terms for the nonlinear term. In fact, Cahn and Hilliard [3] used such a nonlinear term under a second derivative as in our equation, but one that was not homogeneous, which represented a model for the free energy for multi-phase phenomena.

In the convecting frame of the pulse, the pde becomes the heat equation

$$
\partial_{t} \phi=\partial_{x}^{2} F .
$$

One example of $F$ that proves to be stable (under discretization) is

$$
F=\frac{\alpha}{\psi^{2}}\left[\partial_{x}^{2} \psi-\lambda \psi\right],
$$

where $\psi=\phi^{-1}$. Using the chain rule, we have,

$$
\partial_{t} \phi=-\alpha \lambda \partial_{x}^{2} \phi-\alpha \partial_{x}^{2}\left(\partial_{x}^{2} \phi-2 \frac{\left(\partial_{x} \phi\right)^{2}}{\phi}\right) .
$$

The relation to a discrete eigenvalue equation (13) is important for the stability of the methods, as in other solitary wave formulations. The implementation of equation (14) in terms of primitive variables for confinement of convecting vorticity will be termed "VC2". We define these two formulations, (8) and (14) as prototype NSW's for application to convecting vorticity. We define the three terms in equation (14), $F=F_{0}+F_{1}+F_{2}$, where $F_{0}$ and $F_{1}$ are linear, and $F_{2}$ is nonlinear. It should be noted that the role of the second order term $\left(F_{0}\right)$ in equation (14) is different from most popular nonlinear pde's, such as $\mathrm{KdV}$, that harbor solitary waves: In these, the linear (dispersion) term is the "expansion" term, and the "contraction", or "steepener" term is the nonlinear Burgers-like convection: $\left(\partial_{x} \phi^{2} / 2\right)$. Here, the linear second order diffusion $\left(F_{0}\right)$ term acts to contract the pulse and the nonlinear term $\left(F_{2}\right)$ prevents $\phi$ from changing sign and transfers amplitude from large wavelengths 
to small, where the higher order term $\left(F_{1}\right)$ damps it (this wave number transfer and damping is similar to viscous Burgers convection). When equation (12) converges, the pulse relaxes to the form

$$
\phi \rightarrow \phi_{0} \sec h \gamma\left(x-x_{0}\right)
$$

where $\gamma=\sqrt{\lambda}$ and $\phi_{0}$ and $x_{0}$ are arbitrary constants. An important point is that wavelengths longer than the thin features that are to be confined must have a negative diffusive behavior, so that the features remain confined and are stable to perturbations against spreading. This means that $F_{2}$ must be nonlinear: It is easy to show by Von Neumann analysis that a linear combination of terms, for example of second and fourth order, cannot lead to a stable Confinement for any finite range of coefficients: any wavelength that exhibits negative diffusion would eventually diverge. Further, in this formulation, it is important that equation (14) results from an eigenvalue equation (15), which happens if the factor in front of the nonlinear term in (14) is equal to 2.

The appearance of $\phi$ in the denominator of equation (14) makes $F_{2}$ diverge as $\phi \rightarrow 0$. This is what prevents $\phi$ from changing sign (if it is initially single-signed). Since A in equation (9) is conserved, the integral of $\phi$ over any finite region cannot then diverge. In the discretized version defined below, none of the grid values also can then diverge. This ensures realizability if $\phi$ is a physical quantity such as density. Smolarkiewicz [25] also has rearranged the discretized convection equation so that there is such a term in the denominator for this reason. An interesting paper related to similar "negative diffusion" is Ref [39]. This shows the complexity of the algebra for solitary wave equation with general nonlinearities even when just defining the convection speed: Often, the speed must be solved for as part of a nonlinear ordinary differential equation. If a conservative form such as equation (11) is used, however, the mathematics is simple, since the speed is specified.

4.1.2. Discretized representation - Scalar advection. A very robust discretization results from equation (8). As explained above, this equation, however, does not conserve the centroid position. Hence, the convection speed of the centroid of a pulse-like feature will not be exactly correct. However, for a vortex convecting in a flow, the convecting errors will almost completely cancel if the convecting flow is not too slow compared to the induced flow velocity, and the form will be useful since the centroid speed is almost correct [10]. Otherwise, the formulation of equation (14), which conserves speed of the centroid, and hence results in exactly the correct speed, can be used. The centroid preserving formulation of the discretized pde given in equation (14) can be written

$$
\phi_{j}^{n+1}=\phi_{j}^{n}-\frac{\nu}{2}\left(\phi_{j+1}^{n}-\phi_{j-1}^{n}\right)+a \delta_{j}^{2} F_{j}^{n}
$$

where $\delta_{j}^{2} f_{j}=f_{j-1}-2 f_{j}+f_{j-1}, \nu=\frac{c \Delta t}{h}, a=\frac{\Delta t}{h^{2}}, \Delta t$ is the time step, and $h$ is the grid cell size. (A 1-D compact solution, which may span a number of grid cells, is defined in Ref [19].) Separately, we have derived a minimal possible compact solution in Ref [30]. This "subcompacton" consists of only two non zero grid points and, once formed, continues to be so for all times.(For reference, the simple defining equation for this "subcompacton" is (in 1-D)

$$
\phi_{j}^{n+1}=\phi_{j}^{n}-\nu\left(\phi_{j}^{n}-\phi_{j-1}^{n}\right)+\delta_{j}^{2}\left(d_{j}^{n}\right)
$$

where $d_{j}^{n}=-\min \left(\nu \phi_{j}^{n},(1-\nu) \phi_{j-1}^{n}\right)$. This could of course, be put in the form of equation (16). However, the solution is not continuous when $h \rightarrow 0$, and we feel that 
such a scheme would also not lead to smooth solutions in general higher dimensional problems.) Many conventional CFD schemes could be put in the form of equation (16), where F adds a (typically linear) stabilizing dissipation. However, the role of $\mathrm{F}$ is very different here. The Confinement term, $\mathrm{F}$, in our method, is defined as $F_{j}^{n}=\mu \phi_{j}^{n}-\varepsilon \Phi_{j}^{n}$, where $\Phi$ is a nonlinear function of $\phi$ (given below) and $\mu$ is a diffusion coefficient that can model numerical discretization effects in a conventional convection step (we assume physical diffusion is much smaller). For the last term, $\varepsilon$ is a numerical coefficient that, together with $\mu$, controls the size and relaxation time scales of the confined features. For this reason, we refer to the two terms as "Confinement terms". Upon Taylor expansion, we recover the pde given by equation (14) in the fine grid limit, where the pulse size covers many grid cells.

For simplicity, the semi-discrete limit will be taken for the advection (where time is continuous). However, in the limit of small convection time step, or if a number of these "Confinement" steps are taken for each convection step, then the above form will result. The same is true for the wave equation [35].

(An important difference between the VC solitary wave based method and conventional discretization schemes is that VC does not exhibit "ENO" or "WENO" or "TVD" that they aspire to: In the semidiscrete case, the "peak" or maximum value of the amplitude will change periodically by a small amount as the centroid moves across each grid cell, while the sum remains constant. This is required because the sum of the nodal values $\left(\phi_{j}\right)$ is conserved over a discrete set. (Similar small periodic variations are found in other solitary wave ansätze to discretized nonlinear wave equations. These are sometimes termed "wobble".))

We start with advection of a single-signed scalar, $\phi$ :

$$
\partial_{t} \phi=-c \partial_{x} \phi+\partial_{x}^{2}[\mu \phi-\varepsilon \Phi]
$$

or

$$
\phi_{j}^{n+1}=\phi_{j}^{n}-\nu\left(\frac{\phi_{j+1}^{n}-\phi_{j-1}^{n}}{2}\right)+a \delta_{j}^{2}\left(\mu \phi_{j}^{n}-\varepsilon \Phi_{j}^{n}\right) .
$$

There are many possibilities for $\Phi$. A simple class is

$$
\Phi^{n}=\left[\frac{\sum_{l}\left(\tilde{\phi}_{l}^{n}\right)^{-\rho}}{N}\right]^{-1 / \rho}
$$

where we use a modified scalar: $\tilde{\phi}^{n}=\phi^{n}+\tilde{\delta}\left[\operatorname{sign}\left(\phi^{n}\right)\right]$

The above sum is over a set of $\mathrm{N}$ neighboring grid nodes near and including the node where $\Phi$ is computed. The small positive constant $\left(\tilde{\delta} \sim 10^{-8}\right)$ is added with the sign of $\phi^{n}$ to prevent problems due to finite computer precision. Exactly the above term can be used in multidimensional formulations with the sum in equation (20) taken over nearest neighbors. If $\rho=1, \Phi$ is the harmonic mean of $\phi$ on the local stencil. Other forms could also be used: For example, $\rho=\infty$ corresponds to the minimum of the absolute value: For 2-D and 3-D applications, however, such discontinuous solutions, like the "subcompacton" mentioned above, will usually not result in as smooth distributions as continuous ones, and we use only $\rho=1$ or $\rho=2$.

An important feature of equation (19) is that it is first order "Euler" in time and explicit and centered in space. Without the two terms in the last bracket it would be unconditionally unstable. Without the last term $(\Phi)$, it can be stable, but diffusive. 
With $\varepsilon>\mu>0$, it maintains a short convecting solitary wave form permanently or "permanent wave" (for a range of values of $\varepsilon$ ). This is shown in Figure 2 for a pulse propagation with and without WC. Note that the pulse remains stable with WC and is diffusive, even when a higher order method is used without confinement.

The reason for the stability of this formulation is that the harmonic mean $(\rho=1)$ (or some other similar mean) weights the argument with smallest magnitude more highly than others. For a pulse with a single maximum and monotonically decreasing sides, this means that the difference of the two terms in the bracket is always "upwind" toward the maximum. Since the basic scheme is otherwise bidirectional, the result is a stable "convection" towards the maximum from both sides of the pulse.

The two (positive) parameters, $\varepsilon$ and $\mu$, are determined by the two small scales of the computation, $h$ and $\Delta t$, since we want the small features to relax to their solitary wave shape in a small number of time steps and to have an effective support of a small number of grid cells. Thus, even though $h$ may be small, the Laplacian will be large and the local effect also large. At convergence, $\mu \phi-\varepsilon \Phi \approx 0$. The solution to the above equation that vanishes in the far field is then (modulo a small periodic variation as the centroid moves across each cell), $\phi \rightarrow \phi_{0} \sec h\left[\gamma\left(j-j_{0}-\nu n\right)\right]$, where $\phi_{0}$ and $j_{0}$ are arbitrary constants (since equation (16) is translationally invariant and homogenous of degree 1). With propagation in a smooth external field, this relation is still approximately satisfied, as verified by computations and heuristic arguments [27]. (Also, the pde solution of equation (14) has the same form!)

4.2. Vorticity confinement: Extension to multidimensional Euler equations. VC was initially developed for application to thin convecting vortices, including helicopter rotor tip vortex flows [4] [34] [29], as well as vortex/blade [31] [18] [22] and vortex/fuselage interactions [36]. The original form of the method ("VC1") closely conserves momentum and is accurate for most practical flows. It is also used very effectively for modeling small vortical scales in turbulent wakes [15]. However, for convection of thin strong vortex filaments over long distances in a weak external flow fields, it requires a small correction for accurate convection of the centroid trajectory [32] [7] [37]. These issues are the same as discussed for 1-D convection in section 2. To extend VC to all (incompressible) flows, a second form of the method ("VC2") has been developed, which explicitly conserves momentum. It can be seen in Figure 3 (in 2-D), that the $\mathrm{VC} 2$ form, which is the subject of the present study, results in exact centroid convection (to plottable accuracy).

For general unsteady incompressible flows, the governing equations with Vorticity Confinement are discretizations of the continuity and momentum equations, with added terms:

$$
\begin{gathered}
\nabla \cdot \vec{q}=0 \\
\partial_{t} \vec{q}=-(\vec{q} \cdot \nabla) \vec{q}-\frac{1}{\rho} \nabla p+\left[\mu \nabla^{2} \vec{q}-\varepsilon \vec{s}\right]
\end{gathered}
$$

where $\vec{q}$ is the velocity vector, $p$ is the pressure, $\rho$ is the density, and $\mu$ is a diffusion coefficient that includes numerical effects, for example, due to discretization of the first right hand side (convection) term. (We assume that the Reynolds number is large and that physical diffusion is much smaller than the added terms.) For the last term, $\varepsilon \vec{s}$, for the pulse, $\varepsilon$ is a numerical coefficient that, together with $\mu$, controls the size and relaxation time scales of the convecting vortical regions or vortical boundary 
layers where $\vec{s}=-\nabla \times \vec{\omega}$. For this reason, we again refer to the two terms in the brackets as the "confinement terms".

As in the one-dimensional examples, the pair of confinement terms, which represent spreading or positive diffusion, and "contraction", or negative diffusion, together create the confined structures. Stable solutions result when the two terms are balanced. In this way, corrections are made each time step to compensate for any perturbations to the vortical structure caused discretization error in the convection operator, or the pressure correction.

An important point concerning the structure of the "captured" small scale vortex filaments is that the computed flow field external to the vortical regions is not sensitive to the internal structures. Hence in our computations, it is not sensitive to the parameters $\varepsilon$ and $\mu$, over a wide range of values. For example, a 2-D, isolated, thin, concentrated physical vortex will evolve to an axisymmetric configuration [16] [17]. Further, even a rapidly rotating non-symmetric isolated configuration will be approximately axisymmetric when averaged over a short time [31]. Then, it is well known that the induced flow external to an axisymmetric two-dimensional vortex core is independent of the vortical distribution, and hence will not depend on $\varepsilon$ and $\mu$ as long as the core is thin (and the filament curvature is large, so that the flow is approximately two-dimensional in a plane normal to the filament). Hence, even in 3$\mathrm{D}$, we apply $\mathrm{VC}$ only in planes normal to the direction of $\vec{\omega}$ so that the above argument is valid. Therefore, we expect that the issues involved in setting these parameters will be similar to those involved in setting numerical parameters in many conventional incompressible (and compressible) computational fluid dynamics schemes, such as artificial dissipation in many shock-capturing schemes. Further, for turbulent wake flows, preliminary studies suggest that $\varepsilon$, even if it is constant throughout the flow field, can be used to parameterize finite Reynolds number effects, since it controls the intensity of the smallest resolved vortical scales [15].

4.3. Discretization. For incompressible flow, both Confinement terms are nonzero only in the vortical regions even if $\mu$ and $\varepsilon$ are not, since both the diffusion term and the "contraction" term depend on vorticity and vanish outside those regions. Thus, even if there is a second order isotropic numerical diffusion associated with the convection operator, and the diffusion operators are only second order, outside the vortical regions the resulting accuracy of these terms can be third or fourth order, since this diffusion is just the negative curl of the vorticity (for incompressible flows). i.e,

$$
\nabla^{2} \vec{q}=-\nabla \times \vec{\omega} \rightarrow 0 .
$$

This is a very important property of $\mathrm{VC}$, since it is based on a rotationally invariant form. Some other schemes, which attempt to contract velocity gradients, use "dimensional splitting". These contract the velocity gradient even when there is no vorticity and must be artificially "limited". Thus, VC can effectively "convert" a low order numerical solution to a higher order one by "sweeping away" diffusion error (appearing as vorticity) into the vortex core.

The incompressible fluid dynamic equations for the $\mathrm{VC}$ formulation are spatial discretizations of

$$
\vec{\nabla} \cdot \vec{q}=0
$$

and (in discrete time),

$$
\vec{q}^{n+1}=\vec{q}^{n}-\Delta t \vec{q} \cdot \vec{\nabla} \vec{q}+\Delta t \nabla^{2} \mu \vec{q}^{n}+\Delta t \vec{\nabla} \times\left(\varepsilon \vec{w}^{n}\right)
$$


(where $n$ denotes the time step), or an equivalent form:

$$
\vec{q}^{n+1}=\vec{q}^{n}-\Delta t \vec{q} \cdot \vec{\nabla} \vec{q}-\Delta t \vec{\nabla} \times\left(\mu \vec{\omega}^{n}-\varepsilon \vec{w}^{n}\right)
$$

where $\vec{\omega}_{l}^{n}=\vec{\nabla} \times \vec{q}_{l}^{n}$ and

$$
\vec{w}^{n}=\frac{\vec{\omega}^{n}}{\hat{\omega}^{n}}\left[\frac{\sum_{l}\left(\hat{\omega}_{l}^{n}\right)^{-1}}{N}\right]^{-1},
$$

where $\hat{\omega}_{l}^{n}=\left|\vec{\omega}_{l}^{n}\right|+\delta$. As in section 2, we could expand equations (26) and (27) in a Taylor series and get a stable pde in a multidimensional version. In equation (26), the same difference operator acts on $\vec{\omega}$ and $\vec{w}$. Also, the second confinement term (equation (27)) involves again, the sum over the stencil which consists of the central node (where $\vec{w}$ is computed) and its neighboring $(N-1)$ nodes, and $\delta$ is again, a small positive constant $\left(10^{-8}\right)$ to prevent problems due to finite precision.

To see the effect of $\mathrm{VC} 2$, we take the curl of equation (26). We then get a transport equation for vorticity. For example, in two dimensions,

$$
\partial_{t} \omega=-\vec{q} . \nabla \omega+\nabla^{2}[\mu \omega-\varepsilon \Phi(\omega)]
$$

where $\Phi(\omega)$ is the harmonic mean of $\omega$. This equation, including the confinement terms, is exactly a multi-dimensional, rotationally invariant generalization of the onedimensional scalar advection equation (11) shown to be effective (in a plane normal to $\vec{\omega}$ ). Of course, near a vortex core, the discretized solution can still reflect the four-fold symmetry of the grid. This effect, however, vanishes rapidly away from a vortical region, since the solution of the pde is a stable solitary wave. Further, as explained above and in Ref [10], the rotating flow around a vortex core actually allows a simpler discretization of scalar convection (equation (28)), compared to an axisymmetric convecting passive scalar distribution, due to cancellations as the core rotates.

Equations (27) and (28) involve harmonic means. As explained, these are chosen to weight the small values in the stencils more heavily. This term vanishes when any of the values of its argument vanishes, preventing creation of values of opposite sign (for a range of parameters). Using $\mathrm{VC} 2$, the total vorticity (circulation) in a region surrounding a vortex is conserved. This means that the vorticity cannot diverge due to this term, since the maximum absolute value cannot be greater than the absolute value of sum when all values have the same sign. (This is also a property of the one-dimensional scalar advection example.)

There are a large number of alternative forms that can give similar results, in addition to the harmonic mean. We believe that the term should have a smooth algebraic form, to give smooth results, which would be more appropriate for multidimensional applications than forms involving logic functions, such as "minmod", which are sometimes used in 1-D "limiter" schemes.

An alternative example to harmonic mean would be a geometric mean. This should result in a Gaussian distribution instead of a hyperbolic secant. This alternative has not yet been investigated.

4.4. Dynamics of attached boundary layers. It was shown in section 2 for constant pressure that $\mathrm{VC}$ will prevent spreading of the BL due to numerical diffusion. The same is true for the full Euler equations with general external pressure 
distribution as long as the $\mathrm{BL}$ is attached. The BL will then evolve along the surface, but can be approximated with only several grid cells (in the normal direction). It is only necessary to force the velocity to zero just below the surface.

4.5. Separation. The initiation of separation (at least in thin laminar BL's), has been shown to result from a singularity in the tangential velocity gradient. This leads to a large "spike" in normal pressure, followed by one in normal velocity, which causes ejection of fluid. Though this was first shown in 2-D (stretched) grid computations, it can easily be shown that it is also true in a BL "confined" to only several grid cells (in the normal direction). The effective "contraction" velocity from section 2 then serves as an "escape velocity", for fluid ejection from the BL. After ejection, the fluid stream is again captured by $\mathrm{VC}$ (automatically) and forms a convecting vortex sheet. If this sheet subsequently becomes unstable and rolls up, it then is again captured (automatically) and forms a vortex filament. In this way, the BL evolves through all three equilibrium stages of $\mathrm{VC}$.

5. Examples of computed results of VC involving BL's. Many results have already been shown using the initial version of Vorticity Confinement, VC1. Some of these results are included in Ref [33]. In this paper, we discuss the use of $\mathrm{VC} 2$ for the problems discussed in section 1, which include approximations for attached flow and BL separation from regions of high curvature and/or large adverse pressure gradient. Flows with separation from smooth surfaces with turbulent boundary layers will, of course, depend on the details of the boundary layer flow. Currently, conventional models with a number of empirical parameters discretized on very fine grids must be employed. We believe that $\mathrm{VC} 2$ can serve as an inexpensive, efficient "starting basis" for these flows, since the simple VC method itself results in qualitatively reasonable flows with no additional parameters or extra terms, on relatively coarse grids. If additional dynamical details must be included, these can be included as simple perturbations following other work using solitary waves [2].

5.1. Vortex induced separation. Wall-vortex interactions are crucial in many engineering problems. They are responsible for regeneration in turbulent BL's. Further, in many flows such as rotorcraft flows, upstream wakes interact with following rotor blades producing unsteady blade loading and noise. Similar phenomena are also observed in turbomachinery. A simplified model in 2D to demonstrate this interaction involves a rectilinear convecting vortex above an infinite wall placed in a stagnant fluid. The adverse pressure gradient induced by the primary vortex creates a secondary recirculation zone attached to the surface of the wall, which will eventually grow and separate. The unsteady interactions between the wall and the outer region will then lead to the abrupt eruption of this zone. The opposite circulation of the secondary eddy induces an upward motion to the primary vortex. These features can be seen in Figure 4(a). The velocities at two stations, one through the primary vortex and the other through the secondary vortex are shown in Figure 4(b).

This computation involved only a $128 \times 128$ uniform grid, with a vortex of opposite sign to the BL, initially placed 9 grid cells above the wall. A time sequence of the vorticity contours is shown in Figure 5. Also, it can be observed that the vortices and the BL only span 4-5 grid cells. A lot of experimental work has been done in the past [14] [6] demonstrating this eruption phenomenon. The qualitative results from VC2 match quite well with results of much more expensive computations (see Figure 6). An important point is that, unlike vortex particle schemes [9], the computation does not involve placing special markers on the surface with extra logic to determine their 
strength.

This computation reproduces the salient features of the separation dynamics, which the $\mathrm{VC} 2$ dynamics is "attracted" to, without specifying any solution details before the computation.

Another demonstration is shown, which exhibits the same salient features, involving vortex pair interaction with a cylinder. Initially, the vortices convect under their mutual flow only. The boundary layer induced by the vortex pair separates as they approach the boundary. Secondary eddies are created, which, like the above case, have the opposite circulation compared to the BL. It can be seen that the motion of the primary vortices is strongly affected. The vorticity contours at different times are shown in Figure 7. It can be seen that the same qualitative results are produced in a physical water table experiment done by the first author (with Emory Puskas) (see Figure 8).

5.2. Turbulent flat plate boundary layer. Another problem of interest is the flow over a flat plate aligned with a grid plane, as shown in Figure 9(a). A trip wire with 4 grid cells height is used to perturb the flow. It can be seen that the amplitude of this spanwise perturbation grows downstream and the velocity becomes three dimensional, as expected. Also, the streamwise streaks in the boundary layer in Figure 9(b), then become turbulent. The RMS velocity in the streamwise direction is shown Figure 10 at 4 streamwise stations. Upstream of the perturbation (Figure $10(a))$, the flow is laminar and the boundary layer is thin and smooth. The trip wire is introduced 16 grid cells downstream of the inlet, which causes the flow to speed up as shown in Figure 10(b). It can be seen that the boundary layer thickness increases as the flow becomes turbulent. This demonstrates that a full, time dependent turbulent $\mathrm{BL}$ can be simulated using very few grid cells, if $\mathrm{VC}$ is used, and that the results are quite realistic.

The above problem is repeated on a flat plate inclined to a grid plane at an angle $\left(22.5^{\circ}\right)$. This demonstrates the ability of $\mathrm{VC} 2$ to accommodate complex bodies with no fine adaptive or surface conforming grids. The methodology used to immerse the object is described in section 2. First, a flat plate without a tripwire is computed. The isosurfaces of Vorticity magnitude are shown in Figure 11(a). It can be seen that the boundary layer stays thin. A cross-sectional snapshot in Figure 11(b) shows the stable and thin boundary layer, which is only 3 grid cells wide. Now, a trip wire is introduced 16 grid cells downstream of the inlet. The time sequence of the vorticity magnitude isosurfaces is shown in Figure 12. It can be seen in Figure 13 that the trip wire introduces streamwise streaks, which become turbulent, as in the aligned case above. Another simple demonstration for flow over a cylinder is shown in Figure 14. This shows the turbulent wake in the downstream. With conventional methods, finer adaptive grids (typically cylindrical) are required to capture the wake. Using VC, the simulation used a $64 \times 64 \times 32$ Cartesian grid with a computing time of 1 hour on a single processor.

6. Conclusion. There are five main points that should be emphasized in describing VC:

1. Conventional numerical schemes diverge when dissipation (eddy-viscosity) becomes negative, but $\mathrm{VC}$ can remain stable.

2. The above property of $\mathrm{VC}$ results from the use of a nonlinear "contracting" term, which forms a solitary wave.

3. Unlike schemes that use limiters (typically along separate coordinate axes) which involve first derivatives of velocities, $\mathrm{VC}$ uses an intrinsically rotation- 
ally invariant formulation. This involves vorticity, which is the only possible combination of first derivatives of velocity that is rotationally invariant for incompressible flow (in 2-D planes normal to a vortex). This results in a much more efficient way to treat small scale vortices.

4. With conventional schemes on feasible grids, the apparent stochastic behavior of the small vortical scales in physical turbulence must be explicitly put in "by hand". This is probably true if the only deterministic turbulence model terms used are dissipative, like conventional eddy viscosity, which are intrinsically stabilizing. However, VC behaves as an intrinsically negative dissipation at the small scales and can generate natural, chaotic flows. This allows us to take a more natural approach with fewer artifices.

Acknowledgements. Most of the VC work referenced was funded by Dr. Tom Doligalski at ARO and by Dr. Frank Caradonna at AFDD. Recent work was funded by ARO under Dr. Frederick Ferguson. Helpful conversations with Dr. Frank Caradonna and Dr. Arje Nachman, as well as Professor Stan Osher, are acknowledged.

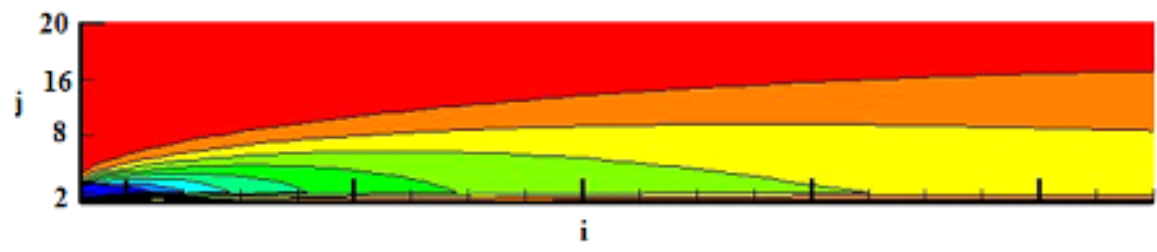

(a) Without Confinement

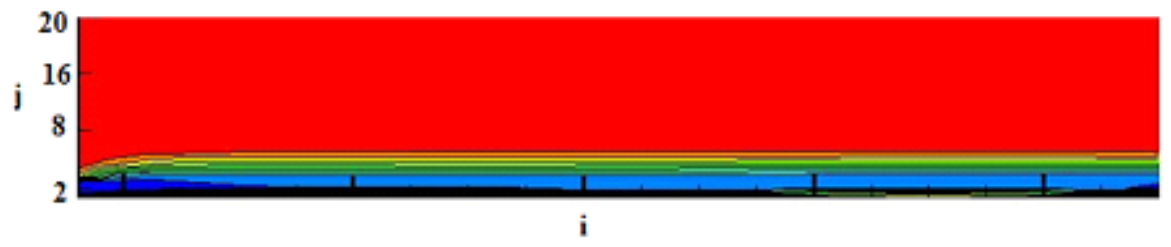

(b) With Confinement

FIG. 1. Boundary layer above a flat plate with and without VC2

\section{REFERENCES}

[1] M. B. Esfe, H. B. Esfe, And M. M. JAfarian, Comparison of various compressible vorticity confinement methods and development two new confinement parameters, Journal of Applied Fluid Mechanics, 5:3 (2012), pp. 89-98.

[2] A. R. Bishop, J. A. Krumhansl, and S. E. Trullinger, Solitons in condensed matter: A paradigm, Physica D: Nonlinear Phenomena, 1:1 (1980), pp. 1-44.

[3] J. W. CAhn AND J. E. HilliaRd, Free energy of a non-uniform system. i. interfacial free energy, Journal of Chemical Physics, 28:2 (1958), pp. 258-267.

[4] M. Costes And G. Kowani, An automatic anti-diffusion method for vortical flows based on vorticity confinement, Aerospace Science and Technology, 7:1 (2003), pp. 11-21.

[5] M. Costes, T. Renaud, B. Rodriguez, and G. Reboul, Application of vorticity confinement to rotor wake simulations, International Journal of Engineering Systems Modelling and Simulation, 4 (2012), pp. 102-112.

[6] T. L. Doligalski, C. R. Smith, And J. D. A. Walker, Vortex interactions with walls, Annual Review of Fluid Mechanics, 26 (1994), pp. 573-616. 


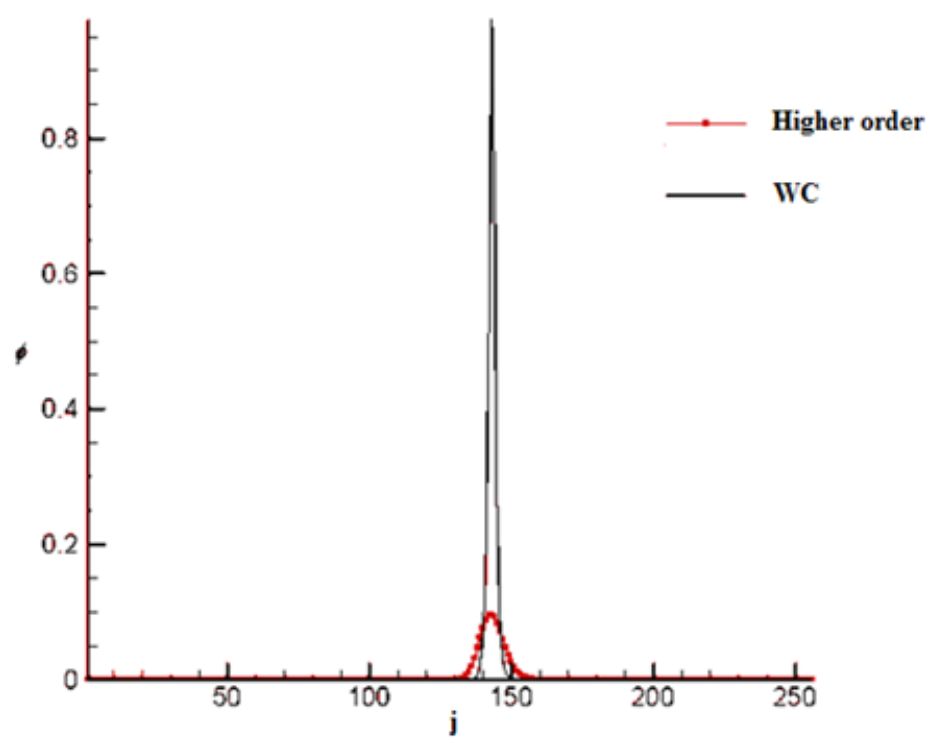

FIG. 2. 1D pulse convergence

[7] S. HAAS, Computation of trailing vortex flow over long distances using vorticity confinement, Master's thesis, Institut fur Luft-und Raumfahrt, RWTH Aachen, Germany, 2003.

[8] A. HaRTEN, The artificial compression method for computation of shocks and contact discontinuities iii, self-adjusting hybrid schemes, Mathematics of Computation, 32:142 (1978), pp. 363-389.

[9] K. HenryK And M. Ziemowit, Investigation of unsteady vorticity layer eruption induced by vortex patch using vortex particles method, Journal of Theoretical and Applied Mechanics, 45:4 (2007), pp. 785-800.

[10] J. Steinhoff, M. FAn, AND L. WAng, Convection of concentrated vortices and passive scalars as solitary waves, Journal of Scientific Computing, 19 (2003), pp. 457-478.

[11] N. Lynn J. Steinhoff And L. Wang, Implicit Large Eddy Simulation: Computing Turbulent Flow Dynamics, chapter Large Eddy Simulation Using Vorticity Confinement. Cambridge University Press, 2006.

[12] T. JANG, H. KIm, J. BAE, J. SEO, And J. Noh, Multilevel vorticity confinement for water turbulence simulation, The Visual Computer, 26 (2010), pp. 873-881.

[13] J. Katz And A. Plotkin, Low Speed Aerodynamics, Cambridge University Press, New York, NY, 2001.

[14] A. Luton, S. Ragab, And D. Telionis, Interaction of spanwise vortices with a boundary layer, Phys. Fluids, 7 (1995), 2757.

[15] N. Lynn, Investigation of Vorticity Confinement as a High Reynolds Number Turbulence Model, PhD thesis, University of TN, 2007.

[16] M. V. Melander, J. C. MCWilliams, and N. J. Zabusky, Axisymmetrization and vorticitygradient intensification of an isolated two-dimensional vortex through filamentation, Journal of Fluid Mechanics, 178 (1997), pp. 137-159.

[17] A. Misra And D. Pullin, A vortex-based stress model for large-eddy simulation, Physics of Fluids, 9 (1997).

[18] R. Morvant, K. Badcock, G. Barakos, and B. Richards, Aerofoil-vortex interaction simulation using the compressible vorticity confinement method, in "29th European Rotorcraft Forum", 2003.

[19] P. P. Rosenau, J. M. Hyman, and M. Staley, Multidimensional compactons, Phys. Rev. Lett., 98 (2007), 024101.

[20] P. L. Panton, Incompressible flow, Wiley-Interscience, John Wiley and Sons, New York, NY, 1996.

[21] R. M. Rennie R. Kelly, A. Jemcov and E. Jumper, The aero-optical environment around a helicopter computed using the compressible vorticity confinement method, in " $44 \mathrm{TH}$ AIAA 
Plasmadynamics and Lasers Conference", AIAA, 2013.

[22] G. G. Barakos R. Morvant, K. K. Badcock, Aerofoil-vortex interaction using the compressible vorticity confinement method, AIAA journal, 43:1 (2005), pp. 63-75.

[23] M. Robinson, Application of vorticity confinement to inviscid missile force and momentum, in "42nd AIAA Aerospace Sciences Meeting and Exhibit", AIAA, 2004.

[24] L. Rosenhead, The formation of vorticies from a surface of discontinuity, Proc. Roy. Soc. Series A, 134:823 (1931), pp. 170-192.

[25] P. K. Smolarkiewicz, A simple positive definite advection scheme with small implicit diffusion, Monthly Weather Review, 111:3 (1983), pp. 479-486.

[26] J. Steinhoff, Vorticity confinement: A new technique for computing vortex dominated flows, Frontiers of Computational Fluid Dynamics, 1994.

[27] J. Steinhoff, S. HaAs, M. Xiao, N. Lynn, and M. Fan, Simulating small scale features in fluid dynamics and acoustics as nonlinear solitary waves, in "41st Aerospace Sciences Meeting and Exhibit", AIAA, 2003.

[28] J. Steinhoff, N. Lynn, Y. Wenren, M. Fan, and L. Wang, Implicit Large Eddy Simulation: Computing Turbulent Flow Dynamics, Turbulent Flow Simulations Using Vorticity Confinement, Cambridge University Press, 2006.

[29] J. Steinhoff, T. Mersch, and Y. Wenren, Computational vorticity confinement: Two dimensional incompressible flow, in "Proceedings of the 6th Southeastern Conference on Theoritical and Applied Mechanics", 1992.

[30] J. Steinhoff, E. Puskas, S. Babu, Y. Wenren, and D. Underhill, Computation of thin features over long distances using solitary waves, in "13th Computational Fluid Dynamics Conference", AIAA, 1997.

[31] J. Steinhoff and G. Raviprakash, Navier-stokes computation of blade vortex interaction using vorticity confinement, in "33rd Aerospace Sciences Meeting and Exhibit", AIAA, 1995.

[32] J. Steinhoff And Y. Wenren, Computation of generation and convection of aircraft vortices, NASA SBIR Contract NAS1-97068 Final Report, 1997.

[33] J. Steinhoff, Y. Wenren, C. Braun, L. Wang, and M. Fan, Application of vorticity confinement to the prediction of the flow over complex bodies, Frontiers of Computational Fluid Dynamics, pp. 197-226, 2002.

[34] J. Steinhoff, Y. Wenren, T. Mersch, and H. Senge, Computational vortex capturing: Application to helicopter rotor flow, in "30th Aerospace Sciences Meeting and Exhibit", AIAA, 1992.

[35] J. Steinhoff And S. Chitta, Solution of the scalar wave equation over very long distances using nonlinear solitary waves: Relation to finite difference methods, Journal of Computational Physics, 231:19 (2012), pp. 6306-6322.

[36] C. M. WANG, Steinhoff J, AND Y. Wenren, Numerical vorticity capturing for vortex-solid body interaction problems, in "11th AIAA Computational Fluid Dynamics Conference", AIAA, 1993.

[37] Y. Wenren, J. Steinhoff, and R. Robins, Computation of aircraft trailing vortices, SBIR Final Report, 1995.

[38] Westwater, Reports and memoranda. Technical Report 1692, Aeronautical Research Committee, 1936.

[39] Thomas P. Witelski, The structure of internal layers for unstable nonlinear diffusion equations, in "Studies in Applied Mathematics, pp. 277-300, 1996.

[40] T. W. Wright, The physics and Mathematics of Adiabatic Shear Bands, Cambridge University Press, 2002. 


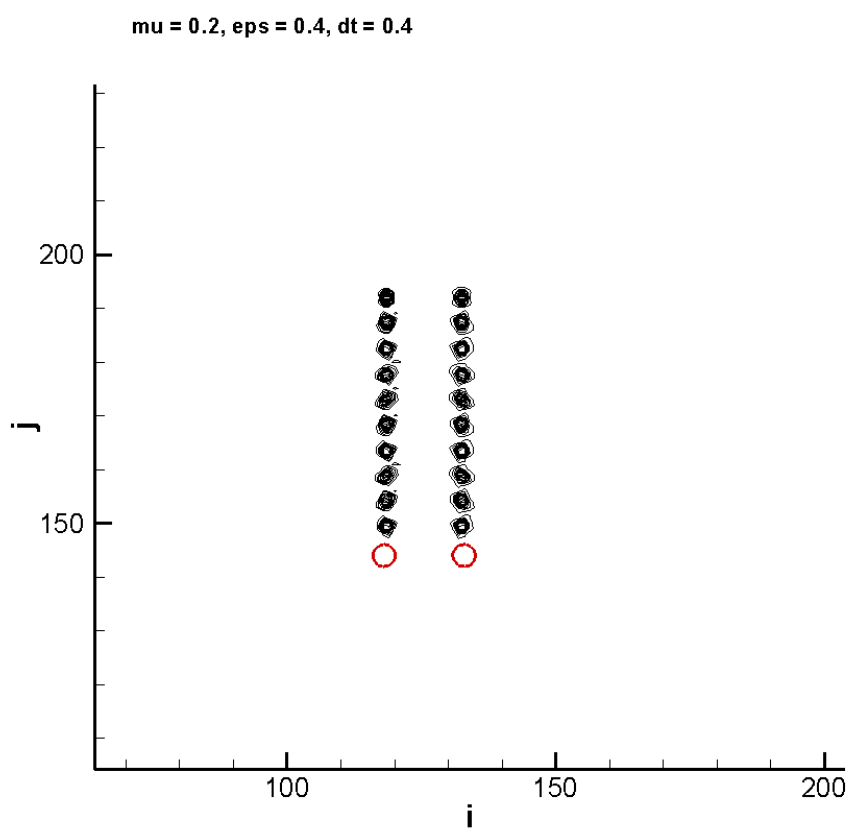

(a) VC1 (black) vs. Experiment (red)

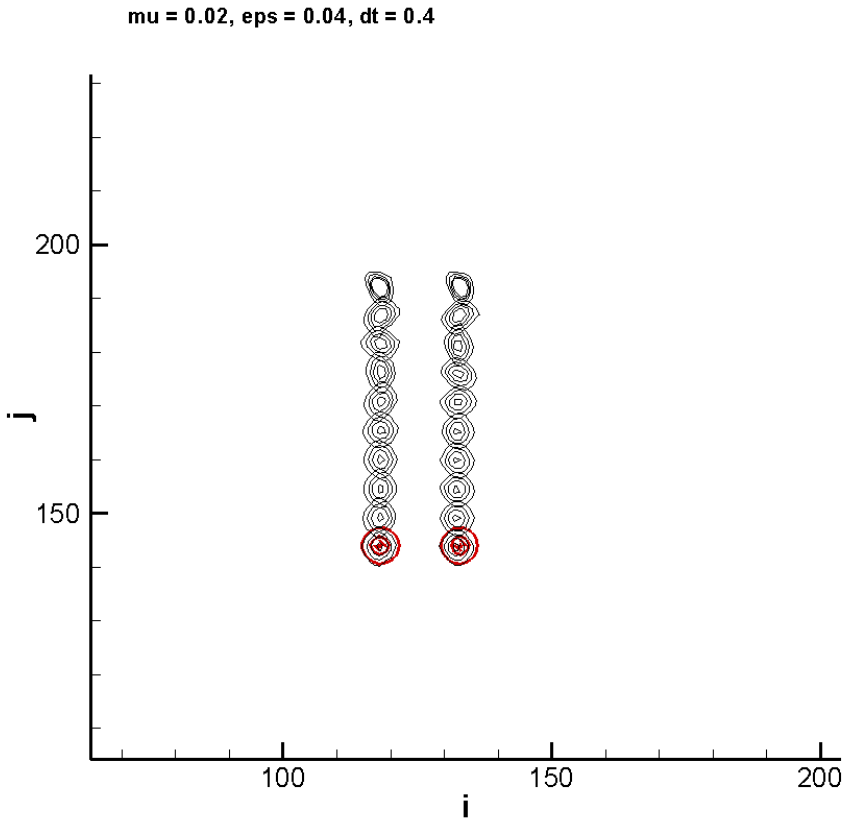

(b) VC2 (black) vs. Experiment (red)

FIG. 3. Vortex centroid convection 


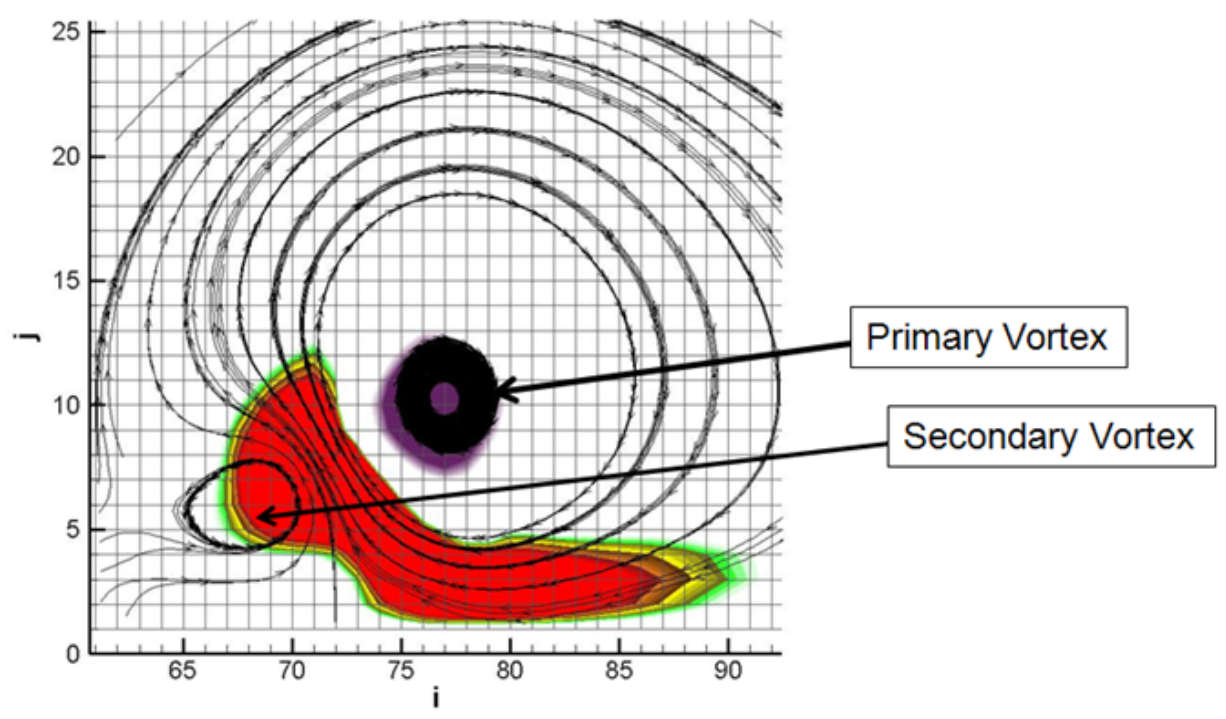

(a) Instantaneous streamlines and vorticity contours
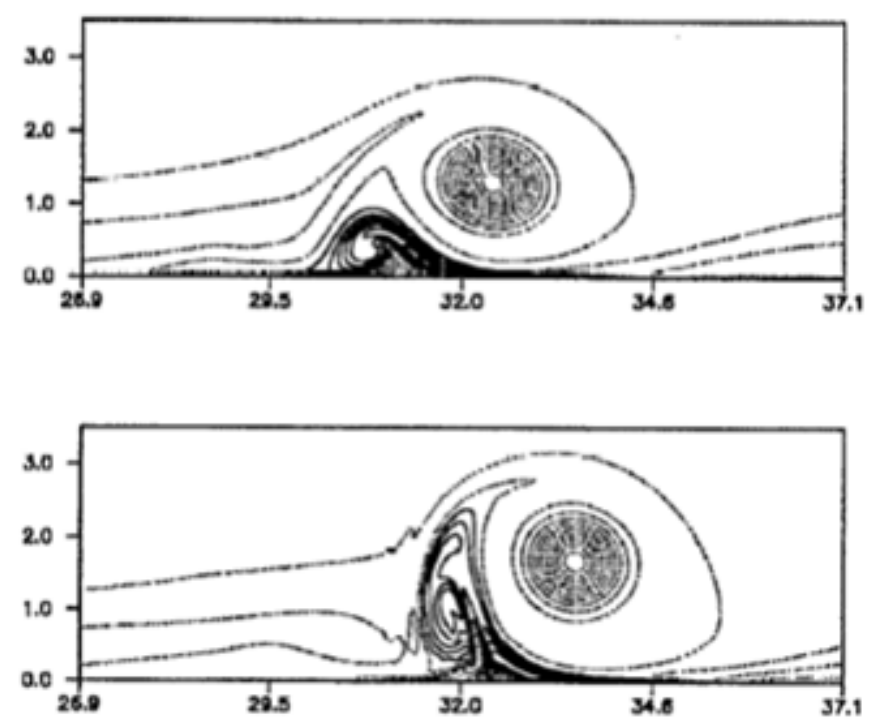

(b) Stream-wise velocity

Fig. 4. Primary and Secondary Vortices 

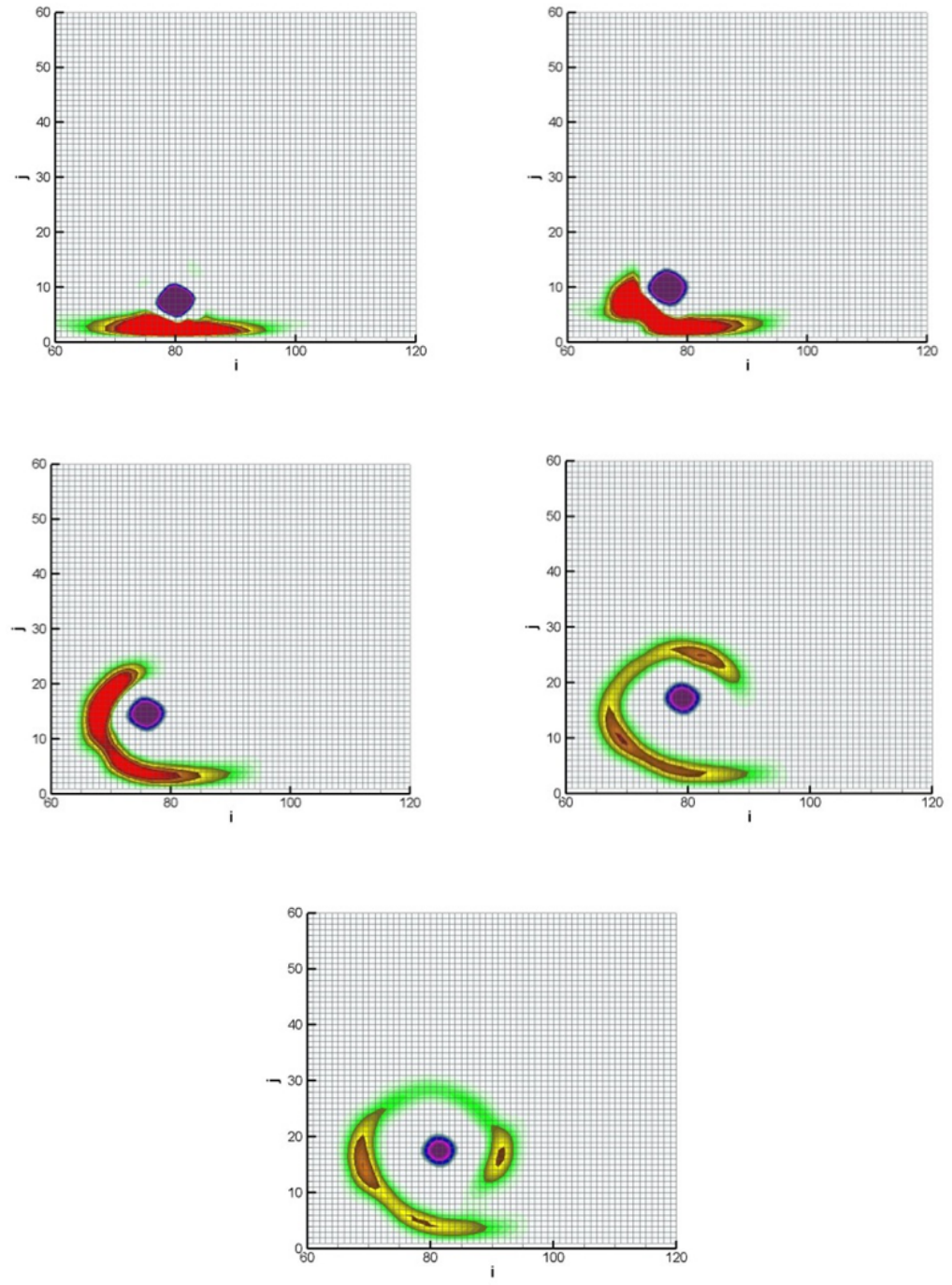

FIG. 5. Time Sequence of Vorticity Contours 

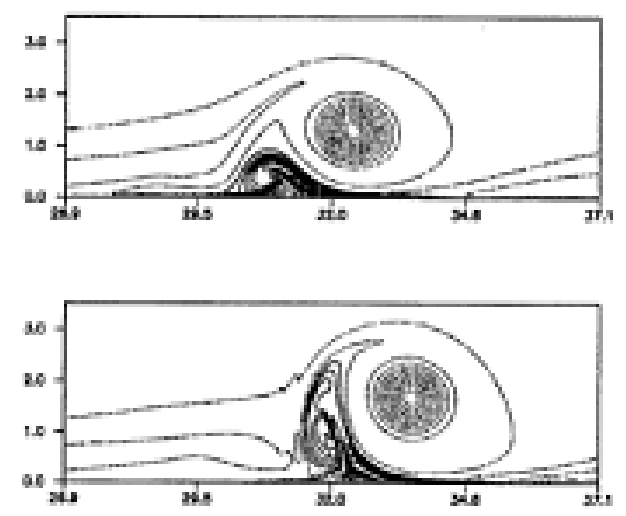

FIG. 6. Vorticity Contours of Primary and Secondary Recirculation Zones from Ref [14]
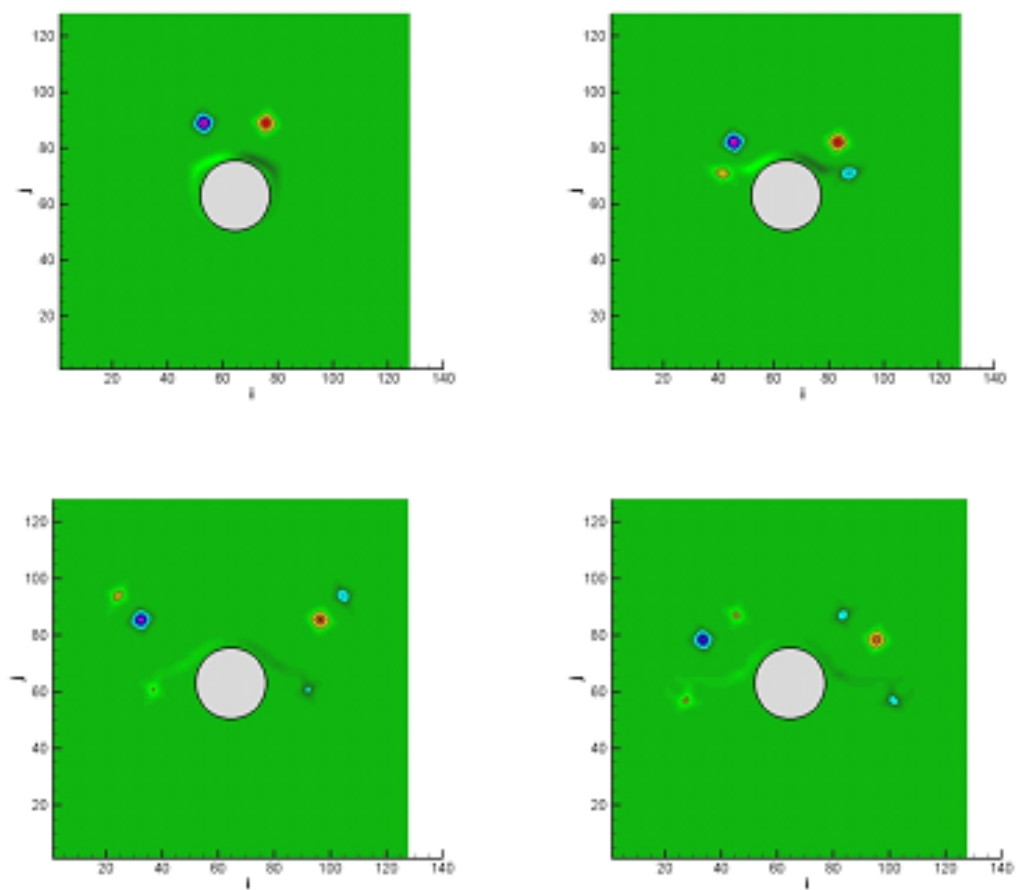

FIG. 7. Vortex Induced Eruption from Cylinder using VC2 

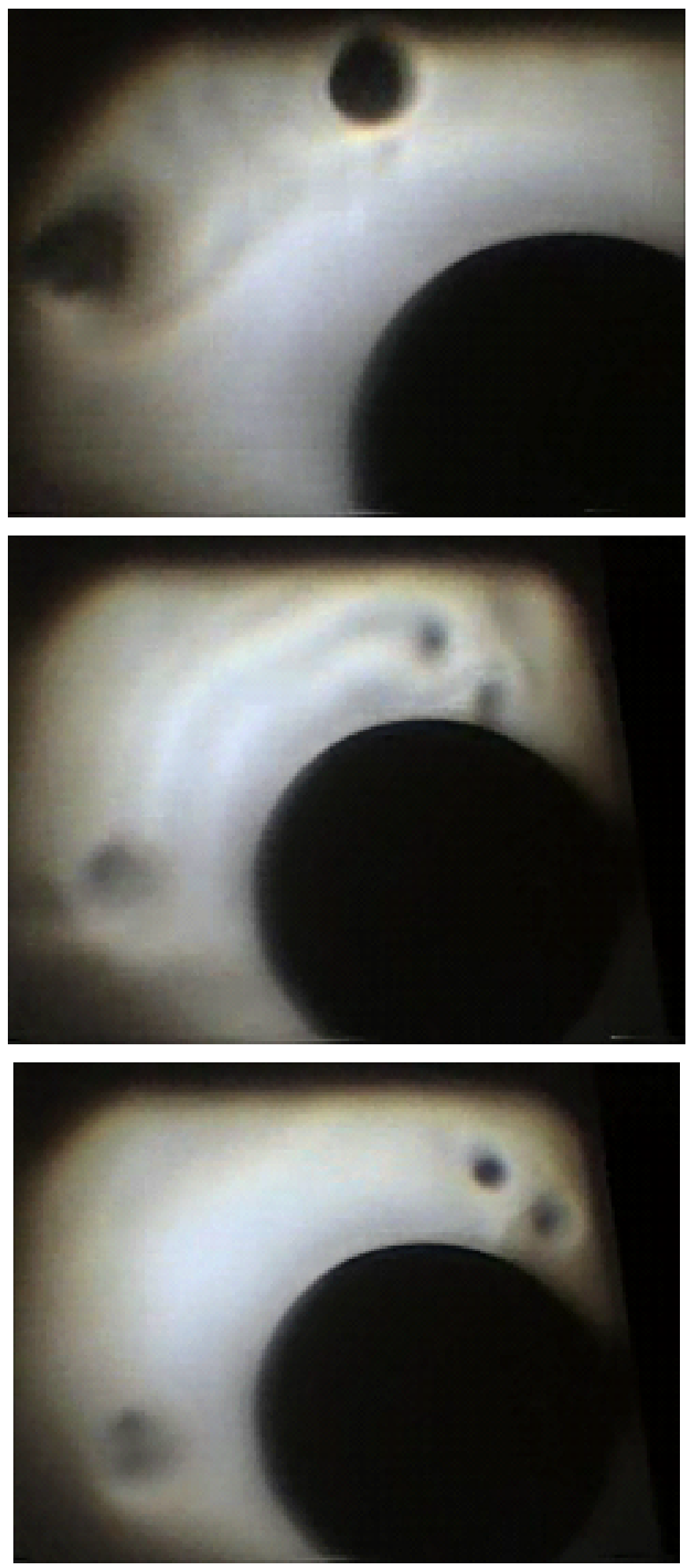

FIG. 8. Vortex Induced Eruption from Cylinder (water table experiment by first author) 


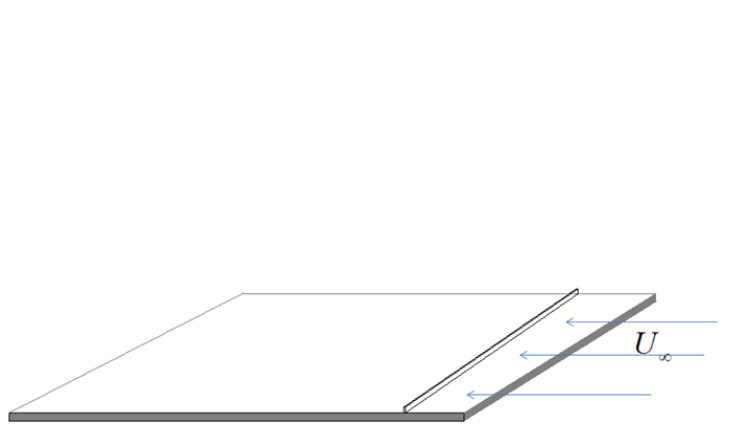

(a) Problem Description

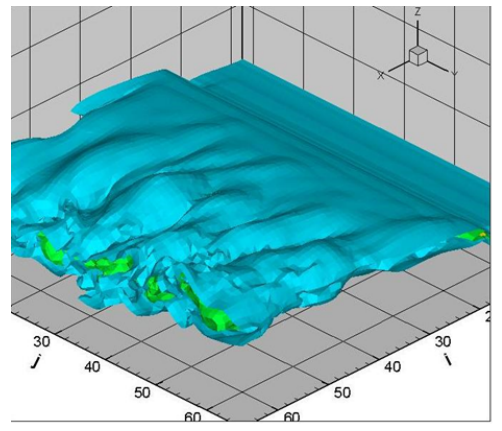

(b) Iso-surfaces of Vorticity Magnitude

FIG. 9. Flow over a flat plate with a trip wire

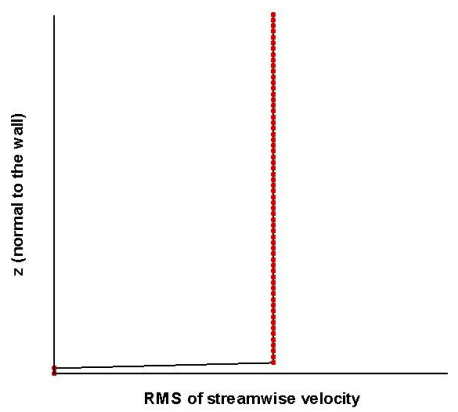

(a)

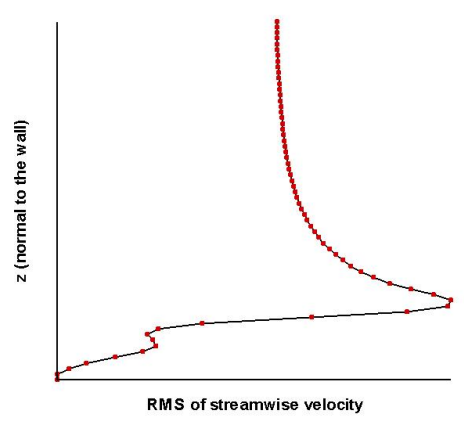

(c)

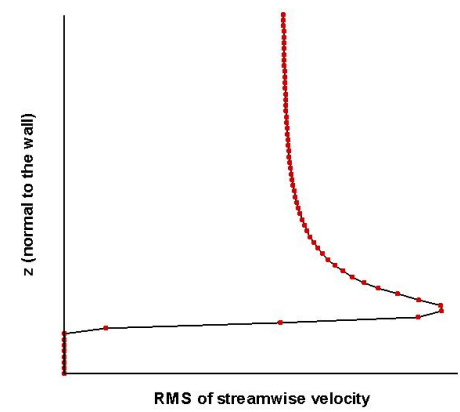

(b)

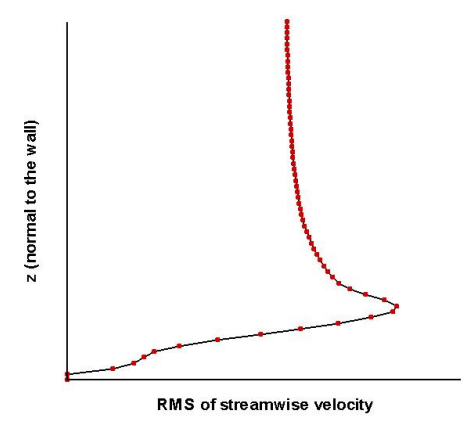

(d)

FIG. 10. RMS Velocity in Streamwise Direction 


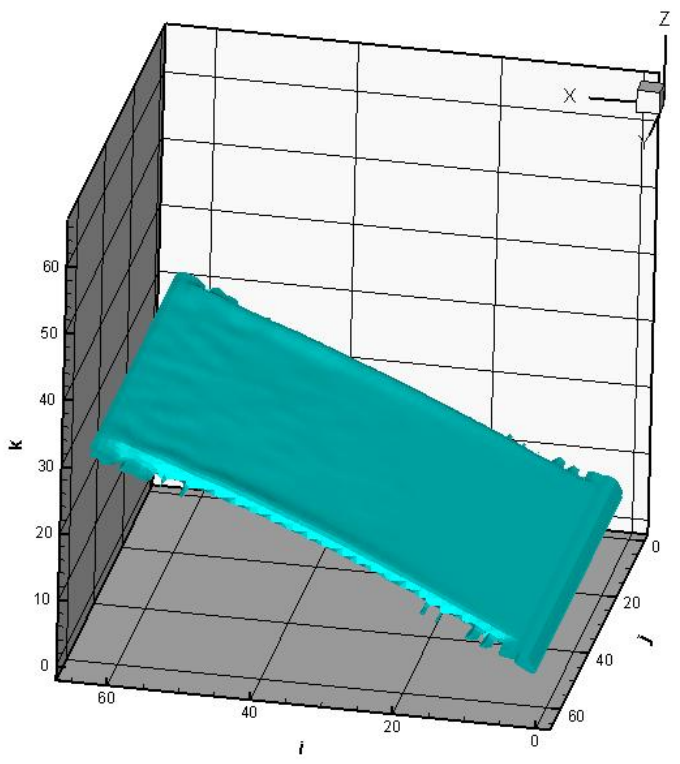

(a) Iso-surfcaes of Vorticity Magnitude

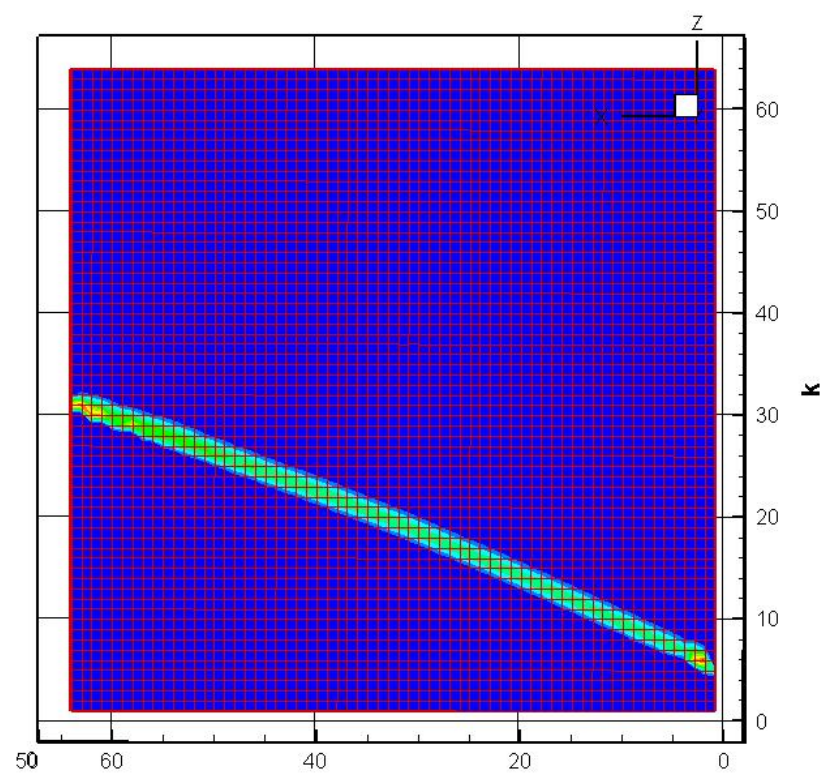

(b) Contours of Vorticity Magnitude on a Cross-sectional Slice

FIG. 11. Flow Over a Flat Plate at an Angle using VC2 


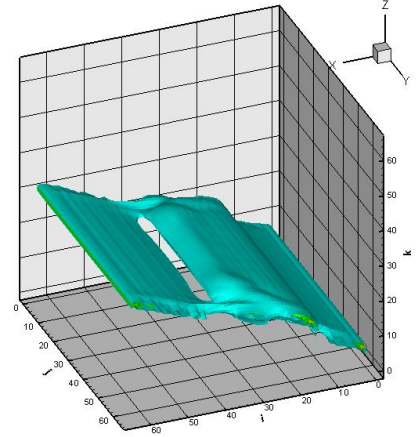

(a)

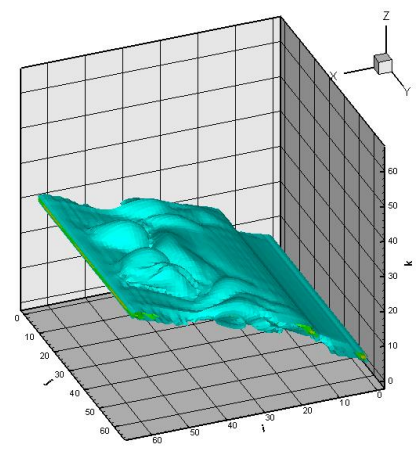

(c)

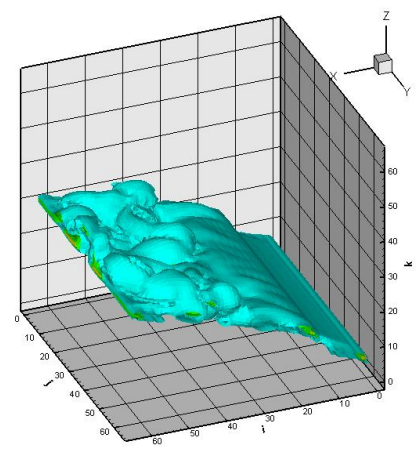

(e)

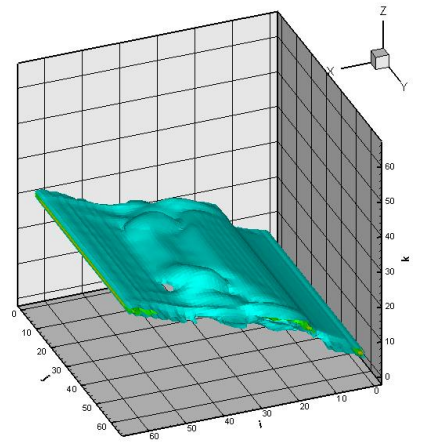

(b)

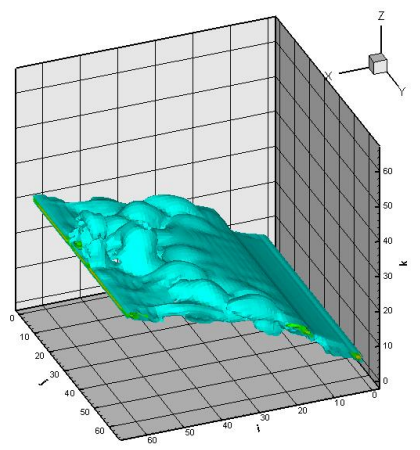

(d)

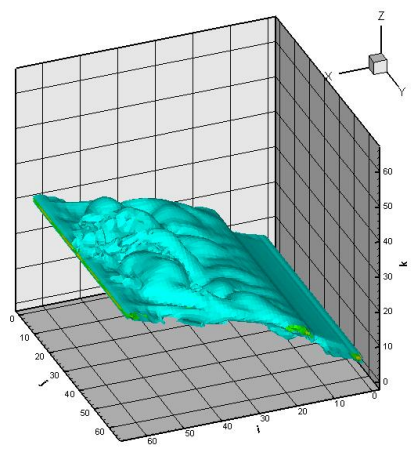

(f)

FIG. 12. Transition to Turbulence above a Flat Plate at an Angle 


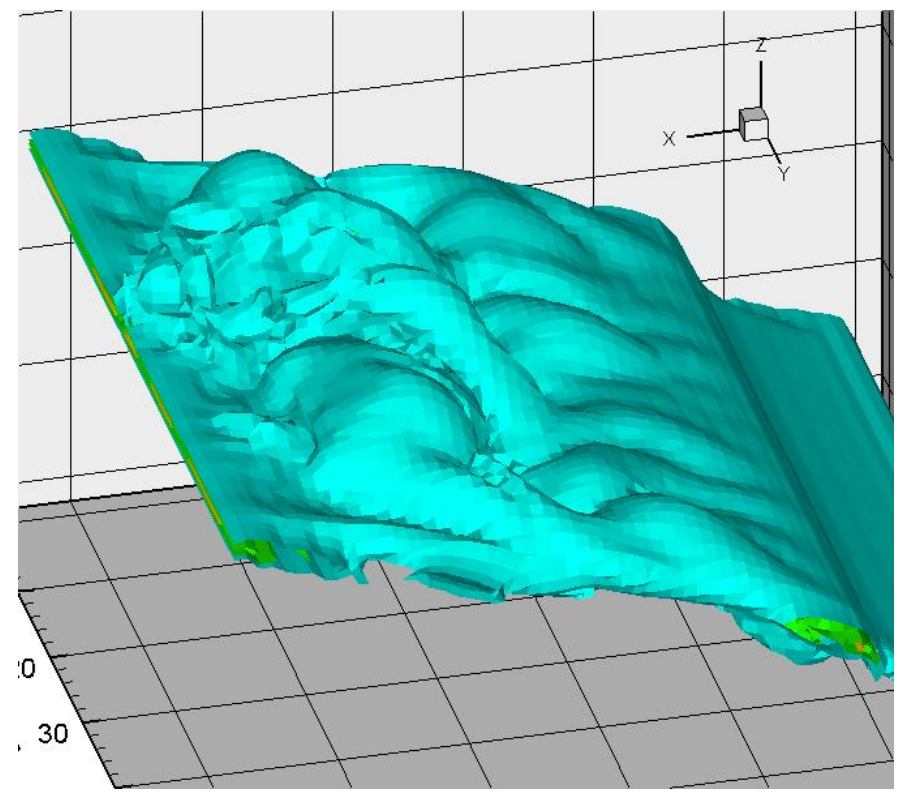

FIG. 13. Streamwise Streaks due to Transition

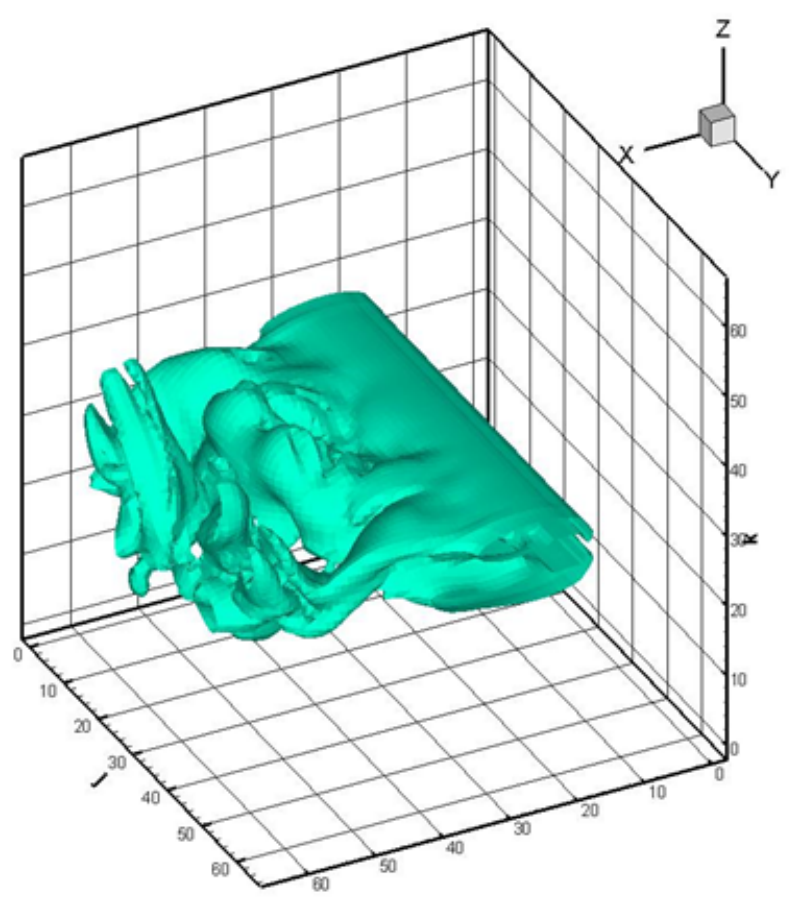

Fig. 14. Boundary Layer Separation 